\title{
Review Article \\ The Effects of Nonpharmacological Treatment on Uremic Pruritus Patients: A Systematic Review
}

\author{
Chiu-Feng Wu, ${ }^{1,2}$ Ya-Chu Hsiao, ${ }^{3}$ and Pi-Chen Ko ${ }^{1}$ \\ ${ }^{1}$ Nursing Department, Ditmanson Medical Foundation Chia-Yi Christian Hospital, Chiayi, Taiwan \\ ${ }^{2}$ Graduate Institute of Nursing, Chang Gung University of Science and Technology, Chia-Yi Campus, Chiayi, Taiwan \\ ${ }^{3}$ Nursing Department, Chang Gung University of Science and Technology, Taoyuan, Taiwan \\ Correspondence should be addressed to Ya-Chu Hsiao; yjshiao@gw.cgust.edu.tw
}

Received 23 January 2015; Accepted 31 May 2015

Academic Editor: Marie-Luise Friedemann

Copyright (C) 2015 Chiu-Feng Wu et al. This is an open access article distributed under the Creative Commons Attribution License, which permits unrestricted use, distribution, and reproduction in any medium, provided the original work is properly cited.

\begin{abstract}
Background. Around 50-90\% of hemodialysis patients develop pruritus. Although studies examining nonpharmacological treatments for itchy skin have been conducted, the conclusions have not been decisive. Purpose. Through a systematic review of the literature, this study aimed to understand nonpharmacological interventions carried out in clinical trials for uremic pruritus and to evaluate and consolidate the information regarding these improvements and their effectiveness. Methods. A literature search focusing on studies published between January 2004 and December 2013 was conducted from 5 electronic databases. After screening based on inclusion criteria and excluding duplicates, nonpharmacological treatments examined in randomized clinical trials were selected for further analysis and synthesis. A modified Jadad scale was used to evaluate the quality of the identified articles. Results. Seven nonpharmacological studies met the inclusion criteria. The interventions to improve uremic pruritus included using emollients, phototherapy, acupuncture, and thermal therapy. Research showed that using emollients, phototherapy, and acupuncture significantly reduces uremic pruritus. Conclusion. Nonpharmacological interventions are effective for hemodialysis patients with pruritus. Emollients were found to provide the most relief compared to the other methods and constitute a readily available and cost-effective intervention to improve pruritus symptoms.
\end{abstract}

\section{Introduction}

Pruritus is an unpleasant feeling of discomfort in the skin. Patients with chronic diseases, such as cancer, uremia, and liver disease, typically have problems with pruritus caused by disease-related factors [1]. Severe pruritus leads to sleep disorders, anxiety, depression, and social dysfunction, affecting the quality of life of patients, worsening prognoses, and increasing the risk of death and the cost of health care [2-5]. Approximately $50-90 \%$ of hemodialysis patients experience difficulties with pruritus $[2,6,7]$.

The specific nerve pathways of pruritus remain unclear. In general, substances that cause pruritus include kinins, serotonin, proteases, neuropeptides, leukotrienes, and other chemicals [8]. Uremic pruritus is caused by systemic metabolic problems. The factors leading to pruritus in dialysis patients can also include xeroderma, abnormal innervation, excessive phosphorus, calcium ions, allergic reactions to dialysis, overly high levels of C-reactive protein, and hyperparathyroidism $[9,10]$. Zucker et al. [11] indicated that $61 \%$ of uremic patients experience difficulty falling asleep because of pruritus, and $44 \%$ have their sleep disrupted by itchy sensations, in some cases leading to emotional anxiety and depression. Pruritus occurs more frequently at night, and the itchiness often influences patients' sleep and moods [3]. Approximately $20-50 \%$ of patients feel that pruritus negatively affects their lives [12].

Clinical treatment for uremic pruritus begins with changing dialysis methods to reduce the production of pruritogens; however, changing the dialysis method has limited effectiveness in improving pruritus [13]. Drug therapy is another common clinical treatment method. Antihistamines, 


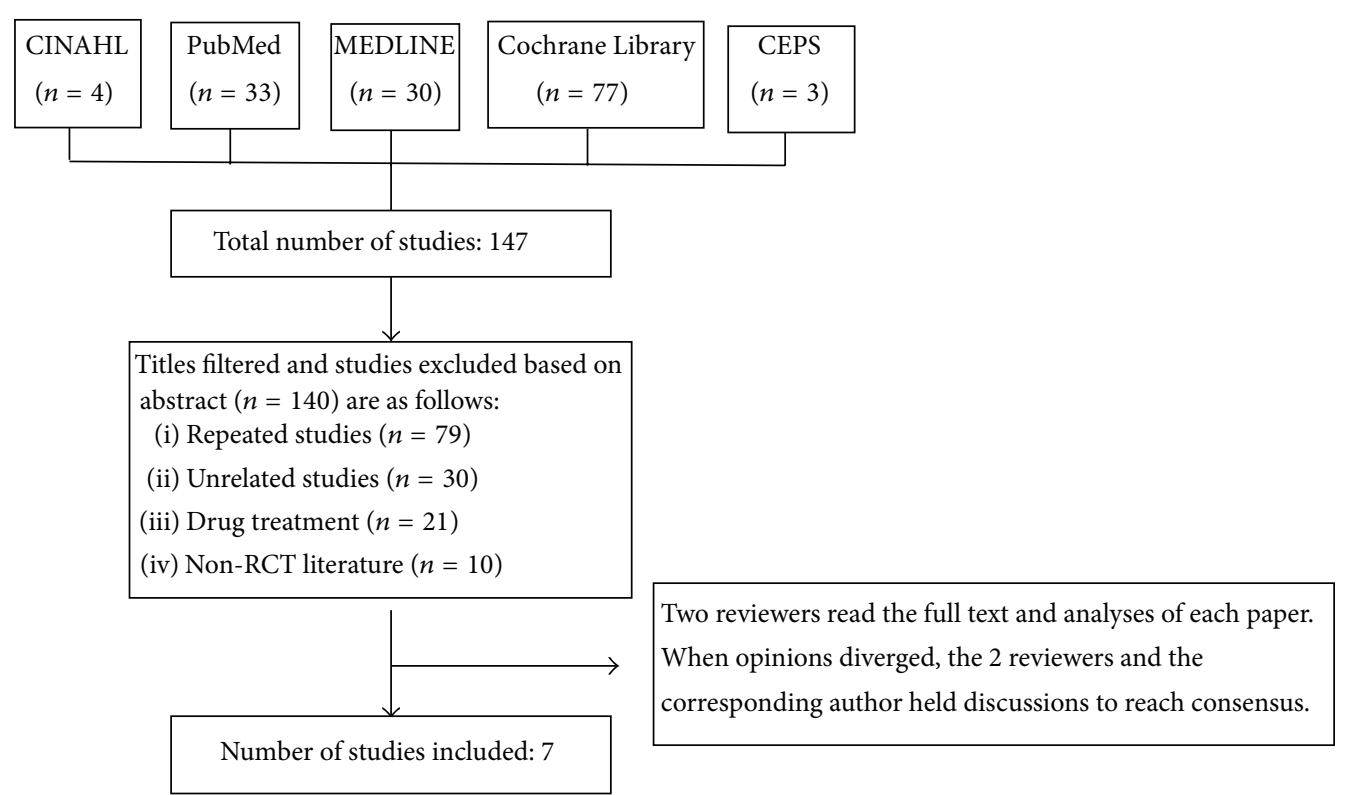

FIGURE 1: Flowchart of finding studies on the effectiveness of nonpharmaceutical treatment for improving pruritus.

capsaicin, opioids, and pramoxine can be administered intravenously, orally, or topically to treat pruritus $[10,13]$. Unfortunately, despite these interventions, pruritus remains a long-term problem for many dialysis patients. Considering the liver and kidney burdens of dialysis patients, the advantages and disadvantages of long-term medication are worth considering. Common alternatives to drug treatment include the application of emollients, ultraviolet irradiation, and acupuncture $[10,13]$, but the intervention methods and results have failed to reveal consistent findings. We therefore conducted a systematic literature review to provide a critical reference for clinical nurses assessing the needs of dialysis patients and to guide patients with pruritus.

\section{Methodology}

2.1. Literature Search Process. We used the empirical search steps proposed by Stillwell et al. [14]. A keyword search using Boolean logic was adopted to search for studies published between 2004 and 2013 in the Cumulative Index to Nursing and Allied Health Literature, PubMed, MEDLINE, the Cochrane Library, and the Airiti Library (the Chinese electronic periodical services). We used patient, intervention, comparison, and outcome as guidelines to establish 3 sets of keywords for the search: (1) "hemodialysis," "renal dialysis," "end-stage renal disease," or "uremic"; (2) "treatment," "therapy," "cure," or "medical treatment"; and (3) "pruritus," "skin itch," or "uremic pruritus." The inclusion criteria were as follows: (1) the research participants had to be adults receiving hemodialysis as their renal replacement therapy; (2) the intervention had to be a nonpharmacological treatment; and (3) the study had to be designed as a randomized clinical trial (RCT). The exclusion criteria were as follows: systematic literature reviews, abstracts, case reports, and studies not in Chinese or English. EndNote and manual proofreading were used to remove duplicate studies as well as those that did not involve treatment for uremic pruritus and those that involved surgery. In total, 140 studies were found that used pharmacological treatments for uremic pruritus or used nonpharmacological treatments but were not RCTs. During the data screening process, if the 2 reviewers disagreed about whether an article should be included, they discussed their views with the corresponding author. Finally, 7 studies were selected (Figure 1 depicts the literature search process). Subsequently, the 2 reviewers each read the full text of these studies and performed an analysis and review. During this process, any disagreements between the 2 check reviewers were discussed with the corresponding author in order to reach consensus, thereby increasing the reliability of the results.

2.2. Literature Appraisal Tools. We adopted a modified Jadad scale to assess the quality of the research designs employed by the examined studies. The Jadad scale is sometimes described as a 5-point scale, though there are only 3 questions: (1) Was the study described as randomized? (2) Was the study described as double-blind? (3) Was there a description of withdrawals and dropouts? [15]. The modified Jadad scale has excellent reliability, yielding an intraclass correlation coefficient of 0.9. The scale is used to rate the content of a study based on the following 8 items (each item receives 1 point): (1) whether the study is described as randomized; (2) whether the method of randomization is appropriate; (3) whether the study is described as double- or singleblind (single blinding receives 0.5 points); (4) whether the method of blinding is appropriate; (5) whether withdrawals or dropouts are described (if the reasons are not described, this item receives 0.5 points); (6) whether the assessment 
of negative events is described; (7) whether the statistical analysis methods are described; (8) whether the inclusion criteria and exclusion criteria are described. The maximum score is 8 ; the range of $0-3$ indicates that the quality is low and $4-8$ indicates that the quality is good to excellent [16].

\section{Results}

In this study, we performed a systematic literature review and compiled empirical studies to determine the effectiveness of nonpharmacological treatments for improving uremic pruritus in hemodialysis patients. Inclusion and exclusion criteria were used to select 7 RCT studies. Table 1 shows the title, participants, intervention measures, measurement indicators, primary results, and research quality scores. For each study, we used serial numbers to compare and explain (1) the basic attributes and quality of each study, (2) the assessment instruments, and (3) the effects of intervention.

3.1. Basic Attributes and Quality. The total number of participants was 338 , with the number of patients in each study ranging from 19 to 93 and the average age spanning from 49 to 67 years. The level of severity of pruritus varied from light to severe, with 2 studies on light pruritus (Numbers 1 and 6), 1 study on moderate pruritus (Number 4), 1 study on moderate to severe pruritus (Number 5), and 3 studies on severe pruritus (Numbers 2, 3, and 7). We scored the research quality based on the modified Jadad scale. The studies all received a rating of 6-7, indicating that the quality was good to excellent. All the studies were randomized, and most of them were assigned appropriately. Only 1 study (Number 7) adopted an in-subject control using a split-body biometrological assessment method. Although the limbs on the 2 sides of the body did not have any statistical differences in skin condition prior to the beginning of the experiment, how the right and left sides were selected for cream application was not explained. Thus, Number 7 received a score of 0 for this item. In terms of blind design, 3 studies employed a double-blind design that was appropriate for these studies (Numbers 1, 3, and 7).

3.2. Assessment Instruments. The assessment instruments employed in the 7 studies were generally aimed at testing the severity of pruritus. This was supplemented with tests evaluating the influence of pruritus on the patients' moods, sleep, and quality of life, or by monitoring changes in blood biochemistry values. Overall, the assessment tools can be divided into 4 categories: assessing the severity of pruritus $(n=7)$, assessing the condition of the skin $(n=3)$, monitoring blood biochemistry values $(n=4)$, and other. Pruritus severity was the primary item for experimental evaluation in the 7 studies, and this was performed using the following 4 assessment instruments:

(1) Visual analog scale (VAS). The VAS comprises selfassessment items for patients to subjectively indicate the severity of their pruritus, with scores ranging from
0 (no itch) to 10 (unbearable). The studies that used this scale were Numbers 1, 2, 5, and 7.

(2) Pruritic score. This score simultaneously indicates the patients' pruritus severity and the influence the condition has on their sleep. The instrument is divided into 2 parts. First, pruritus is assessed by taking the product of the pruritus severity and the number of affected locations on the body. The second part addresses the influence the condition has on patients' sleep. The scores from the first and second parts are added to give a total score, where higher scores indicate a more severe effect on the patients' sleep. The studies that used this scale were Numbers 2 and 3.

(3) Uremic pruritus questionnaire. This questionnaire contains 13 items that are used to assess the frequency and severity of patients' pruritus and sleep disorders. Each item is scored from 0 to 4 based on the severity $(0=$ not present and $4=$ extremely severe). Cronbach's alpha is 0.65 . Study Number 5 used this scale.

(4) Itch severity scale. This scale expands pruritus severity to its influence on participants' daily lives with a total of 7 items: frequency, conditions, location, severity, influence on mood, sexual life, and sleep $(0=$ not present and $3=$ severe). It has a content validity index of 0.95 and Cronbach's alpha of 0.79 . The study that used this scale was Number 6.

In terms of skin condition, 3 studies investigated skin dryness, peeling, itching, and scratch marks (Numbers 2, 4, and 7). In these studies, assessments were made by medical personnel or by using equipment. For example, in Number 2, physicians assessed the skin condition based on the severity of dryness and scratches. In Number 7, a skin detector was used to evaluate skin condition. With respect to blood biochemistry values, 4 studies observed changes in blood biochemistry values (Numbers 1, 3, 4, and 5). The "other" category covers emotional disturbances (Number 1), sleep disorders (Number 4), treatment satisfaction (Number 4), and quality of life (Number 7). These variables were typically measured using single items with scoring based on subjective evaluations.

3.3. The Effects of Intervention. Four types of interventions were used to improve the patients' pruritus: the application of nonpharmacological preparations to the skin, phototherapy, acupuncture, and thermal therapy. Among the 7 examined studies, 5 studies effectively improved the patients' pruritus (71.4\%). The most commonly used interventions were nonpharmacological preparations, which were applied to the skin to improve the patients' pruritus. This intervention was used in 4 studies, and the preparations used were an aqueous gel containing aloe vera (Number 1), lotion containing mud from the Dead Sea (Number 4), baby oil (Number 6), and cream containing sericin (Number 7). Among these 4 interventions, only Number 4 reported nonsignificant experimental results. In Number 1, an aloe-containing aqueous gel was applied twice a day, and the patient's pruritus, dryness, and scratches were effectively improved; however, after two weeks, only 


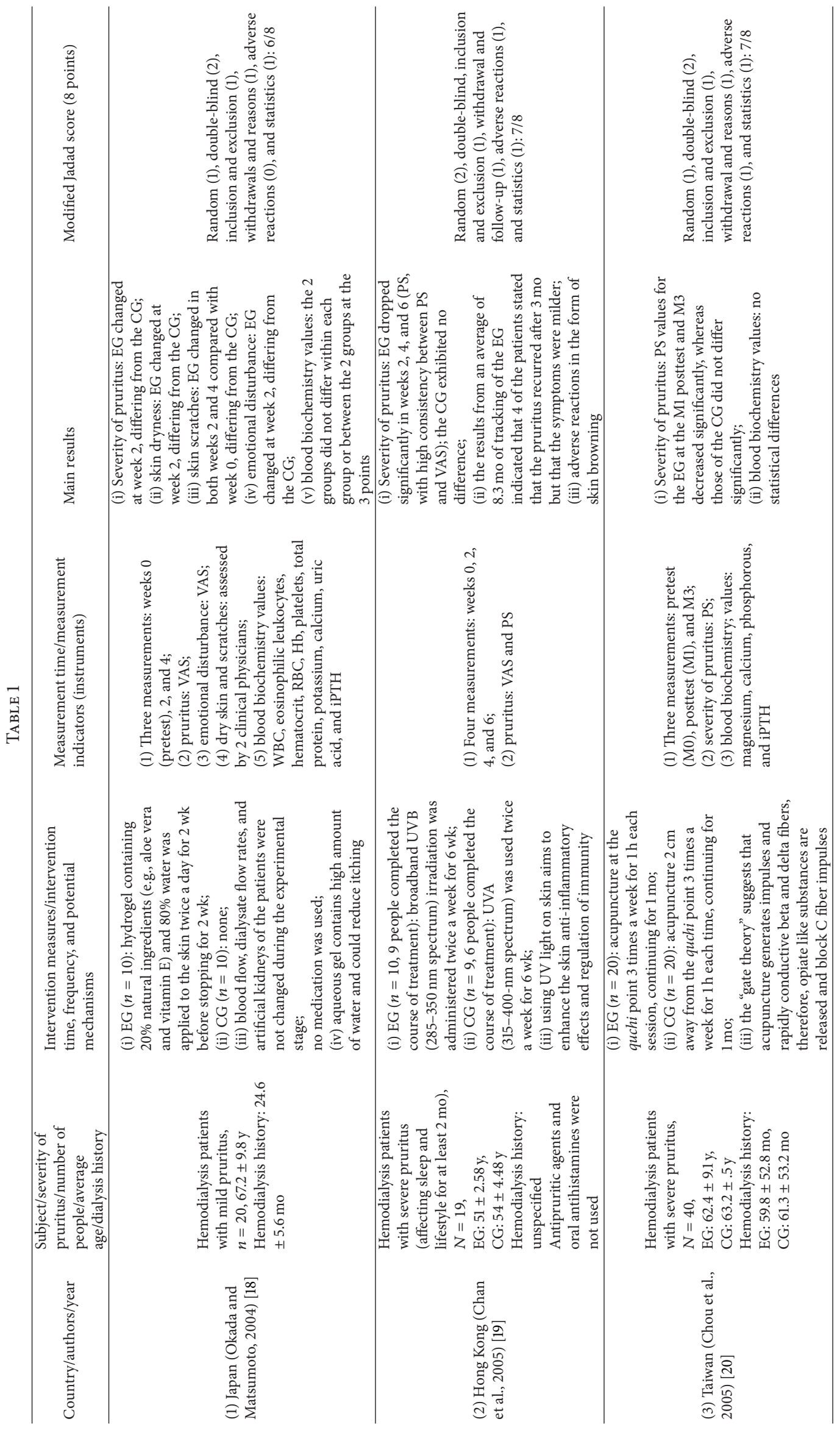




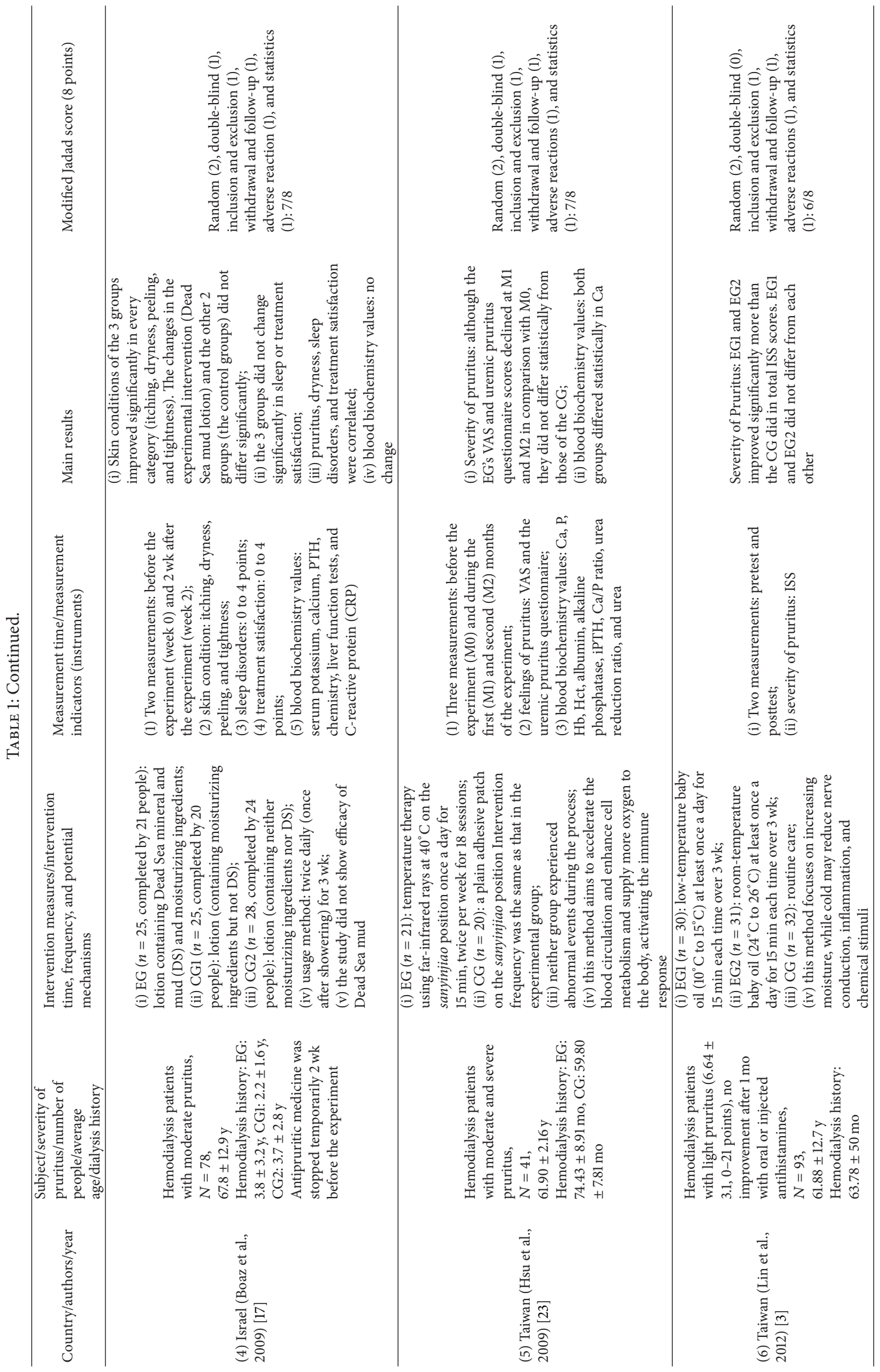




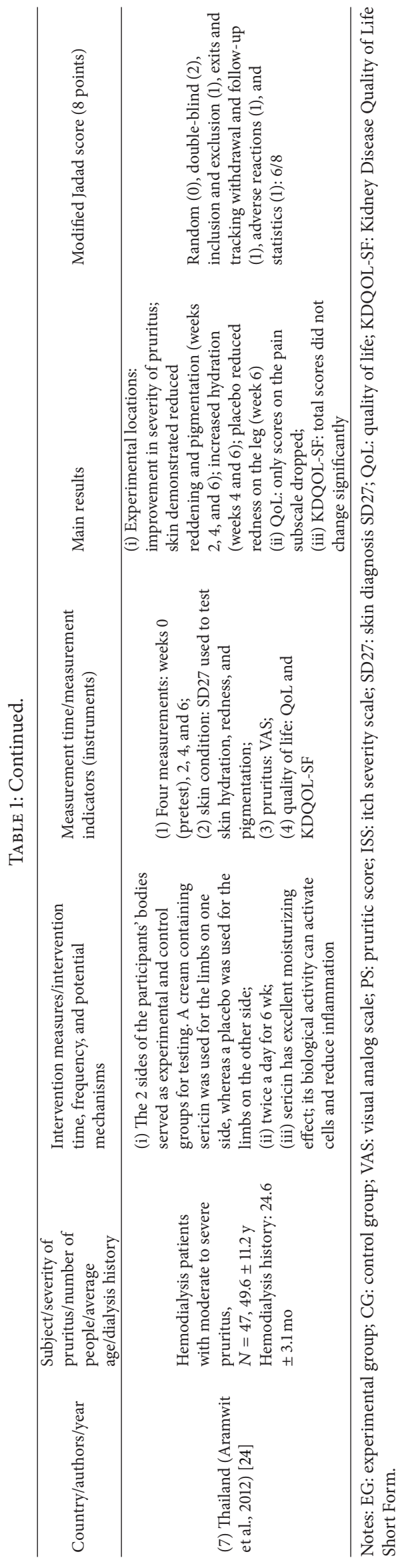


the improvement in the scratches was maintained. Number 6 applied commercially available baby oil once daily. Comparisons between 2 experimental groups, where baby oil was applied at various temperatures $\left(10-15^{\circ} \mathrm{C}\right.$ and $\left.24-25^{\circ} \mathrm{C}\right)$, and a control group, where no special treatment was applied, indicated that baby oil was effective in improving the patients' pruritus, even at different temperatures. Number 7 used silk sericin cream twice a day (once after bathing), effectively improving the patients' pruritus, reducing the skin's redness and pigmentation, and increasing the level of skin hydration.

The second type of intervention measure was phototherapy, which involved the use of ultraviolet (UV) lamps with varying radiation spectra (Number 2). After patients with systemic lupus erythematosus and sensitivity to light were excluded, photo testing was conducted on the research participants prior to the phototherapy interventions to determine the minimal erythema dose (MED) for each participant. The experimental group had their entire bodies (except the eyes and genitals, which were shielded) irradiated with broadband UVB twice a week for 6 weeks. The control group was irradiated with UVA. The experimental group's condition improved from the second week of phototherapy. This improvement persisted throughout the 8.3 months of follow-up.

The third type of intervention measure was acupuncture. The researchers of Number 3 selected the quchi position near the puncture point of the arm fistula of dialysis patients and administered acupuncture for 1 hour during dialysis. The effects of this intervention persisted into the third month following the experiment.

The fourth type of intervention measure was thermal therapy. The researchers of Number 5 applied far-infrared radiation at the sanyinjiao site, and therapeutic effects were observed after radiation. Although the pruritus of the experimental group improved, the improvement did not differ significantly from that observed in the control group. Therefore, the researchers were unable to confirm whether thermal therapy was effective in improving pruritus.

In addition to investigating changes in pruritus (or skin condition), the influences of these 4 intervention measures on the patients' blood biochemistry and quality of life were tested. The patients' existing blood biochemistry values did not influence changes in blood biochemistry values (Numbers 1, 3, 4, and 5); however, thermal therapy (Number 5) influenced the calcium ion concentration in blood serum. In addition, the studies examined the effects of pruritus on patients' daily lives and found that these interventions could simultaneously alleviate the patients' emotional disturbances (Number 1) and reduce the effect pruritus had on the patients' sleep (Numbers 2, 3, and 6).

\section{Discussion}

All 7 studies examined in this systematic literature review were randomized controlled study designs that satisfied our inclusion criteria. Therefore, the integrated conclusions are representative. Emollients were used in 4 studies involving a combined total of 238 participants. Three of those studies found that using emollients can alleviate pruritus. Boaz et al.
[17] indicated that Dead Sea-enriched lotion, moisturizing lotion, and general lotion did not differ significantly in terms of effectiveness in treating pruritus; however, the pre- and posttreatment conditions improved significantly for all 3 groups. Thus, administering emollients on the skin can alleviate pruritus, regardless of whether the emollients contain specific components or differ in temperature. This is a relatively simple, safe, low-cost, and effective method for treating pruritus; however, recommendations should be considered when using emollients. For example, they must be applied regularly and used daily (immediately after patients shower or wash their bodies) to moisturize patients' skin so as to reduce dryness, desquamation, and pruritus. In addition, for the emollients to have a preventative effect, patients should be encouraged to use them before symptoms of pruritus appear [3, 18-20].

Although only 1 study investigated phototherapy and only 1 study investigated acupuncture points, both of these measures were effective in improving pruritus. Following consultation with a dermatologist, Taiwan's National Health Insurance covers the cost of phototherapy for uremic pruritus for dialysis patients. In accordance with previous studies, the researchers of Number 3 administered acupuncture at the quchi position. The relief effects were observed 2 months after ceasing acupuncture therapy. Thus, administering acupuncture to dialysis patients during their dialysis sessions may be a feasible method for alleviating their pruritus condition. In addition, although each experimental design had different follow-up times, which made it difficult to evaluate the longterm effects of the interventions, the study on phototherapy had a relatively long follow-up and observation period. Although the results were inconclusive regarding whether phototherapy has longer antipruritic effects in comparison to the other intervention measures, patients and medical staff are supportive of any intervention measures with long-term effects.

In terms of the influence of the intervention measures on the other variables of pruritus, 6 of the 7 studies collected data regarding the influence of pruritus on patients' sleep disorders. In addition to confirming the results of previous studies that had shown that pruritus affects patients' quality of sleep, the researchers also explored whether mitigating pruritus could alleviate sleep disorders in patients. Four of the 7 studies examined the patients' blood biochemistry characteristics, including their calcium, phosphorus, and parathyroid hormone levels. With the exception of parathyroid hormones (which were reported in 4 of the 7 studies and were high in 2 of those studies), the other values were within relatively normal ranges, which is consistent with the findings of Welter et al. [21]. By contrast, Huang et al. [10] indicated that high calcium, phosphorus, and parathyroid hormone levels cause pruritus, which was also reported by Tajbakhsh et al. [22], except that they reported that parathyroid hormones are unrelated. Therefore, further research may be required to verify the correlation between blood biochemistry characteristics and uremic pruritus.

In this study, we compiled data on the effectiveness of nonpharmacological interventions aimed at alleviating 
uremic pruritus in dialysis patients by systematically reviewing the literature. The results of our study indicated that the quality of the experimental studies was moderate to high; however, the sample size and research instruments in the selected studies were deemed issues worthy of further consideration.

We provide the following recommendations as a reference for future studies. First, all 7 studies had small samples comprising 19 to 93 participants from a single hemodialysis unit. Future studies should consider recruiting participants from multiple hemodialysis units to improve their representativeness or at least recruiting a larger sample to avoid sample bias. Second, most of the studies failed to explain the reliability and validity of their measurement instruments. Future studies could strengthen their findings and improve the reliability of their experimental results by providing this information. In addition, most of the studies employed selfassessment measures to evaluate the patients' pruritus (e.g., the VAS). The possible presence of placebo effects should also be considered. Finally, the degree of blinding and the clarity of the statements in the research design should be controlled as much as possible to reduce the occurrence of the Hawthorne effect.

Limitations. In this study, we systematically reviewed the literature to determine the effectiveness of nonpharmacological interventions in improving uremic pruritus in dialysis patients. Because various types of interventions were used, a meta-analysis could not be performed. Additionally, the examined studies were randomized experimental studies. Consequently, the key findings from other types of studies may have been inadvertently excluded from our analysis. The systematic literature review emphasized data integrity and accuracy. The 7 studies that met our selected criteria were only from limited usable databases. In addition, we analyzed only 7 studies that met our conditions. This limited the results of the discussion and our ability to draw broad inferences from these results.

\section{Conclusion}

Overall, the examined studies indicated that numerous nonpharmacological interventions are effective in alleviating pruritus in dialysis patients. Among the discussed methods, emollients were consistently effective in maintaining adequate skin hydration and alleviating uremic pruritus symptoms; however, only limited inferences can be drawn regarding the other intervention methods (i.e., phototherapy and acupuncture) because only 1 study supported each of these methods. In addition, using emollients does not require additional facilities or special training. The choice of intervention should be made based on patients' habits and what is convenient for them. Using emollients should be feasible and cost-effective for most patients. Although pruritus is not a fatal condition, it affects patients' sleep quality, mood, and general quality of life. The use of emollient preparations appears to be a simple and feasible method for improving pruritus.

\section{Conflict of Interests}

No conflict of interests exists in the submission of this paper, and the paper has been approved by all the authors for publication.

\section{Authors' Contribution}

Chiu-Feng Wu and Pi-Chen Ko conducted database search studies, read the full text of these studies, and performed an analysis and review. Chiu-Feng Wu wrote the paper. Ya-Chu Hsiao read the full text of these studies, performed an analysis and review, and revised the paper.

\section{References}

[1] C. Xander, J. J. Meerpohl, D. Galandi et al., "Pharmacological interventions for pruritus in adult palliative care patients," The Cochrane Database of Systematic Reviews, vol. 6, Article ID CD008320, 2013.

[2] M. Castello and M. Milani, "Efficacy of topical hydrating and emollient lotion containing 10\% urea ISDIN(R) plus dexpanthenol (Ureadin $\mathrm{Rx}$ 10) in the treatment of skin xerosis and pruritus in hemodialyzed patients: an open prospective pilot trial," Giornale Italiano di Dermatologia e Venereologia, vol. 146, no. 5, pp. 321-325, 2011.

[3] T.-C. Lin, Y.-H. Lai, S.-E. Guo et al., "Baby oil therapy for uremic pruritus in haemodialysis patients," Journal of Clinical Nursing, vol. 21, no. 1-2, pp. 139-148, 2012.

[4] H. Rayner, J. Baharani, S. Smith, V. Suresh, and I. Dasgupta, "Uraemic pruritus: relief of itching by gabapentin and pregabalin," Nephron: Clinical Practice, vol. 122, no. 3-4, pp. 75-79, 2012.

[5] B. Wikstrom, "Itchy skin - a clinical problem for haemodialysis patients," Nephrology Dialysis Transplantation, vol. 22, supplement 5, pp. 3-7, 2007.

[6] I. Narita, B. Alchi, K. Omori et al., "Etiology and prognostic significance of severe uremic pruritus in chronic hemodialysis patients," Kidney International, vol. 69, no. 9, pp. 1626-1632, 2006.

[7] L.-J. Lee, B.-J. Kuo, K.-F. Sun, and F.-C. Chen, "Investigation of pruritus and self-management in hemodialysis patients at a medical center in Central Taiwan," Chung Shan Medical Journal, vol. 14, pp. 523-531, 2003.

[8] D. F. Butler, Pruritus and Systemic Disease, Medscape, 2014, http://emedicine.medscape.com/article/1098029-overview.

[9] N. C. V. Melo, R. M. Elias, M. C. M. Castro, J. E. Romao, and H. Abensur, "Pruritus in hemodialysis patients: the problem remains," Hemodialysis International, vol. 13, no. 1, pp. 38-42, 2009.

[10] S.-C. Huang, C.-L. Lin, H.-Y. Lee, S.-C. Yang, T.-I. Ho, and K.-Y. Hung, "Uremic pruritus: associated factors and effects of present treatment," Journal of Taiwan Nephrology Nurses Association, vol. 4, no. 1, pp. 17-24, 2005.

[11] I. Zucker, G. Yosipovitch, M. David, U. Gafter, and G. Boner, "Prevalence and characterization of uremic pruritus in patients undergoing hemodialysis: uremic pruritus is still a major 
problem for patients with end-stage renal disease," Journal of the American Academy of Dermatology, vol. 49, no. 5, pp. 842-846, 2003.

[12] T. G. Berger and M. Steinhoff, "Pruritus and renal failure," Seminars in Cutaneous Medicine and Surgery, vol. 30, no. 2, pp. 99-100, 2011.

[13] J. R. Lugon, "Uremic pruritus: a review," Hemodialysis International, vol. 9, no. 2, pp. 180-188, 2005.

[14] S. B. Stillwell, E. Fineout-Overholt, B. M. Melnyk, and K. M. Williamson, "Evidence-based practice, step by step: searching for the evidence," American Journal of Nursing, vol. 110, no. 5, pp. 41-47, 2010.

[15] A. R. Jadad, R. A. Moore, D. Carroll et al., "Assessing the quality of reports of randomized clinical trials: is blinding necessary?" Controlled Clinical Trials, vol. 17, no. 1, pp. 1-12, 1996.

[16] M. Oremus, C. Wolfson, A. Perrault, L. Demers, F. Momoli, and Y. Moride, "Interrater reliability of the modified Jadad quality scale for systematic reviews of Alzheimer's disease drug trials," Dementia and Geriatric Cognitive Disorders, vol. 12, no. 3, pp. 232-236, 2001.

[17] M. Boaz, L. Shtendik, M. Oron et al., "A randomized controlled clinical trial comparing the efficacy of dead sea mineralenriched body lotion versus two types of placebo in the treatment of cutaneous dryness, itching, peeling and tightness in hemodialysis patients (EDIT)," Nephron-Clinical Practice, vol. 113, no. 3, pp. c169-c176, 2009.

[18] K. Okada and K. Matsumoto, "Effect of skin care with an emollient containing a high water content on mild uremic pruritus," Therapeutic Apheresis and Dialysis, vol. 8, no. 5, pp. 419-422, 2004.

[19] C. M. Chan, C. Y. Leung, T. Y. Lam, K. K. Lo, and K. L. Tong, "A study to evaluate efficacy and safety of broadband UVB in the treatment of uraemic pruritus," Hong Kong Journal of Dermatology and Venereology, vol. 13, no. 2, pp. 69-73, 2005.

[20] C.-Y. Chou, C. Y. Wen, M.-T. Kao, and C.-C. Huang, "Acupuncture in haemodialysis patients at the Quchi (LI11) acupoint for refractory uraemic pruritus," Nephrology Dialysis Transplantation, vol. 20, no. 9, pp. 1912-1915, 2005.

[21] E. D. Q. Welter, R. H. Frainer, A. Maldotti, A. Losekann, and M. B. Weber, "Evaluating the association between alterations in mineral metabolism and pruritus in hemodialysis patients," Anais Brasileiros De Dermatologia, vol. 86, no. 1, pp. 31-36, 2011.

[22] R. Tajbakhsh, H. R. Joshaghani, F. Bayzayi, M. Haddad, and M. Qorbani, "Association between pruritus and serum concentrations of parathormone, calcium and phosphorus in hemodialysis patients," Saudi Journal of Kidney Diseases and Transplantation, vol. 24, no. 4, pp. 702-706, 2013.

[23] M.-C. Hsu, H.-W. Chen, Y.-J. Hwu, C.-M. Chanc, and C.-F. Liu, "Effects of thermal therapy on uremic pruritus and biochemical parameters in patients having haemodialysis," Journal of Advanced Nursing, vol. 65, no. 11, pp. 2397-2408, 2009.

[24] P. Aramwit, O. Keongamaroon, T. Siritientong, N. Bang, and O. Supasyndh, "Sericin cream reduces pruritus in hemodialysis patients: a randomized, double-blind, placebo-controlled experimental study," BMC Nephrology, vol. 13, article 119, 2012. 


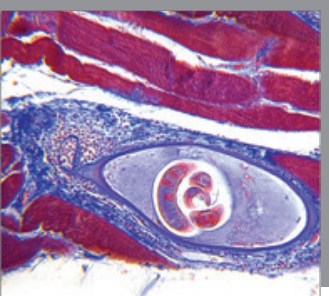

Gastroenterology

Research and Practice
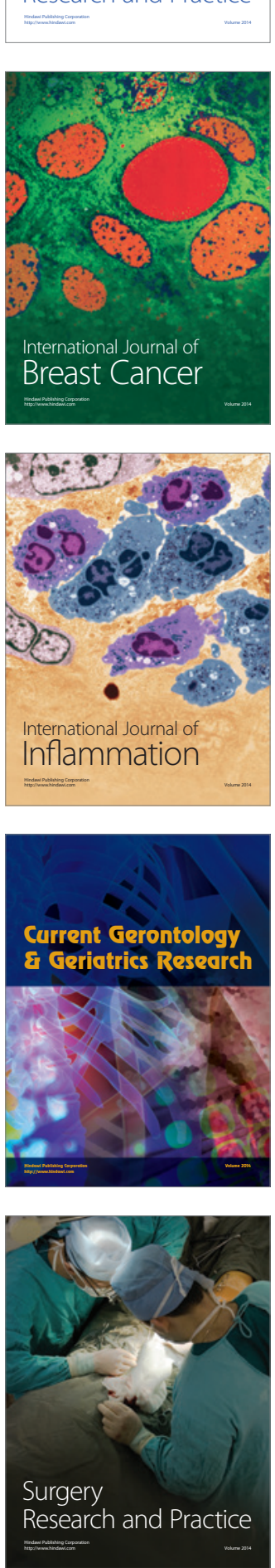

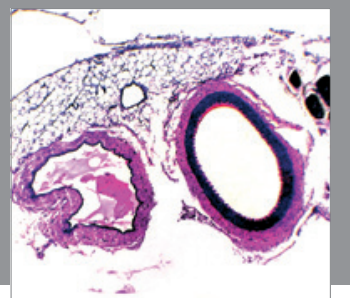

International Journal of Hypertension
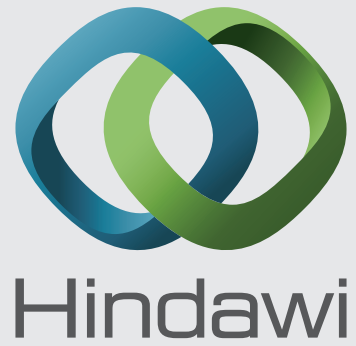

Submit your manuscripts at http://www.hindawi.com
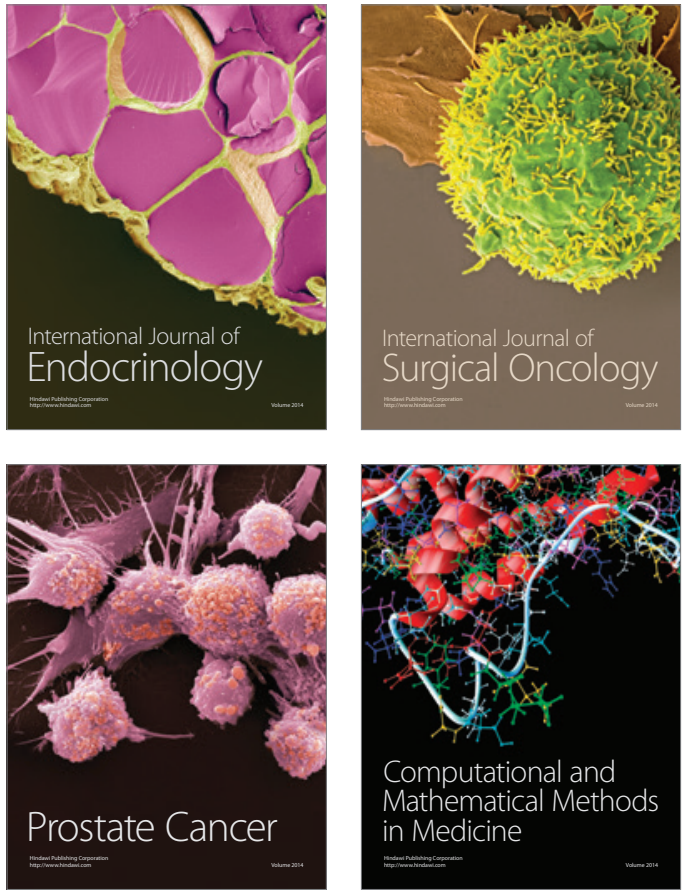
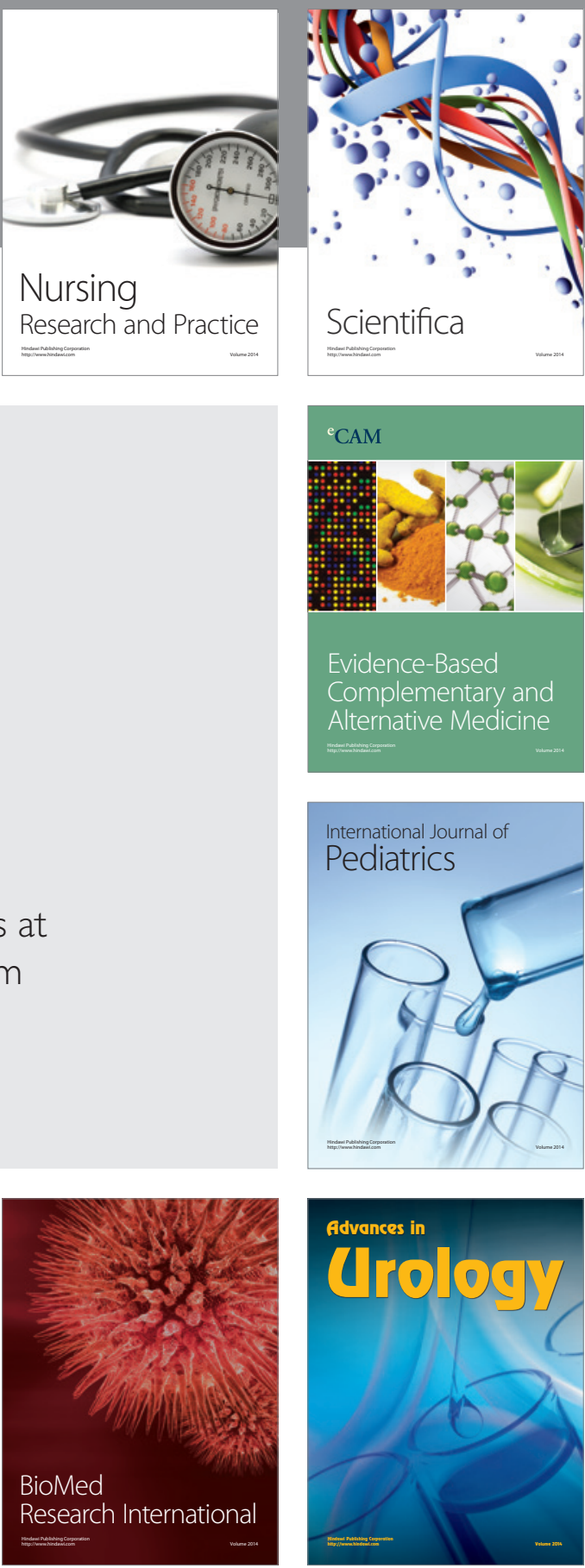

Nursing

Research and Practice

Scientifica

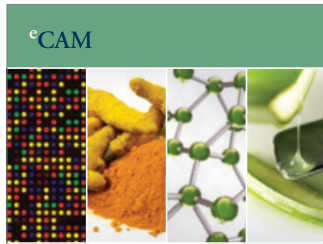

Evidence-Based

Complementary and Alternative Medicine
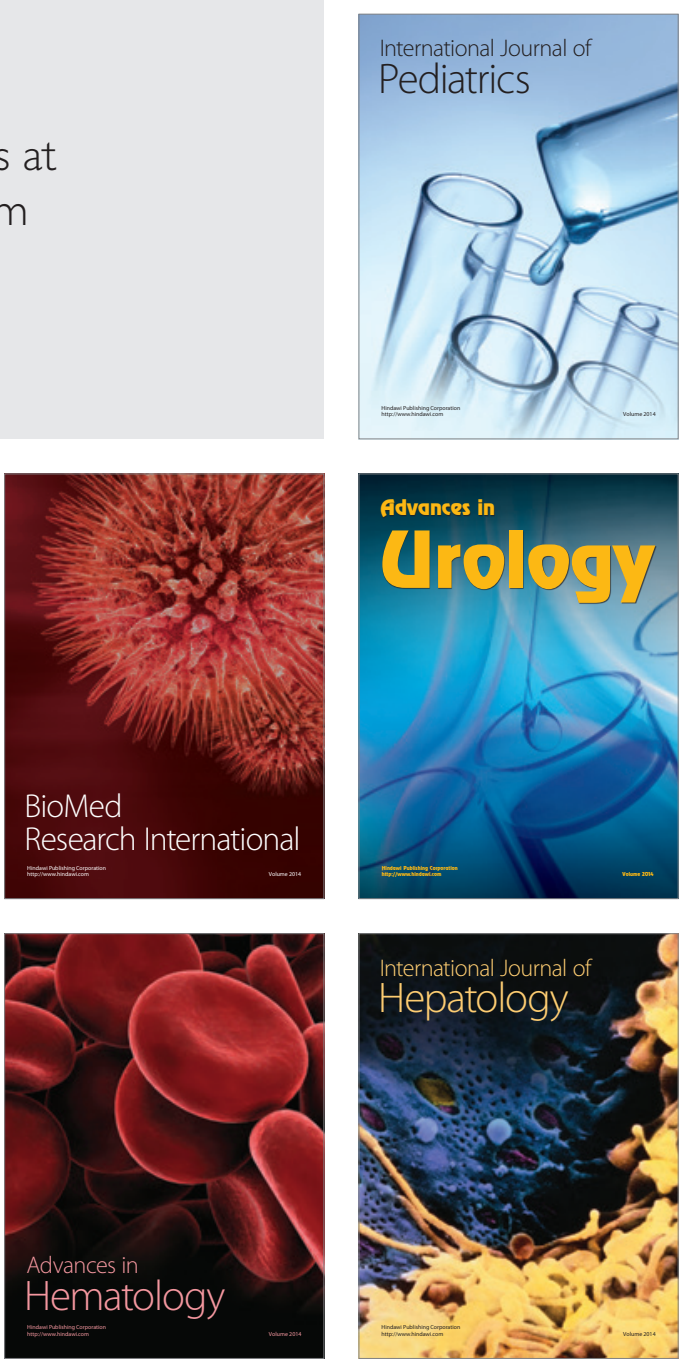